\title{
Modelling and simulation of fluid-structure interactions in human snoring
}

\author{
N.S.J. Elliott ${ }^{\text {a }}$, A.D. Lucey ${ }^{\text {a }}$, M. Heil ${ }^{\text {b }}$, P.R. Eastwood ${ }^{\mathrm{c}}$ and D.R. Hillman ${ }^{\mathrm{c}}$ \\ ${ }^{a}$ Fluid Dynamics Research Group, Department of Mechanical Engineering, Curtin University, \\ GPO Box U1987, Perth WA 6845, Australia \\ ${ }^{\mathrm{b}}$ School of Mathematics, University of Manchester, Oxford Road, Manchester M13 9PL, U.K. \\ ${ }^{\mathrm{c}}$ West Australian Sleep Disorders Research Institute, Sir Charles Gairdner Hospital, \\ Hospital Avenue, Perth WA 6009, Australia
}

Email: n.s.j.elliott@curtin.edu.au

\begin{abstract}
Snoring noise is generated by vibration of the soft tissues of the upper airway, principally those that form the back of the roof of the mouth (the soft palate) and its conical extension (the uvula). In addition to discord with bed partners, snorers are at much greater risk of obstructive sleep apnoea. This sleep-related breathing disorder is characterised by episodic upper airway obstruction with accompanying sleep disruption and consequent excessive daytime sleepiness, as well as an elevated risk of accidents and cardiovascular disease. The instability that leads to flow-induced oscillations characteristic of inspiratory snoring in the human upper airway may be modelled using a cantilevered flexible plate in a mean channel flow. However, the cantilever in existing models strictly only captures the dynamics of the uvula. In a more complete model, these dynamics will be augmented by their interaction with the motions of the soft palate-itself a flexible structure of higher effective stiffness-from which the uvula extends.

To investigate how the elasticity of the soft palate affects uvula motion and their combined susceptibility to flow-induced oscillation a modification is made to the standard model. In a one-dimensional cantilevered flexible plate we allow thickness to vary as a function of length, thus effecting local changes in inertia and flexural rigidity. The overall cantilever therefore comprises a section representing the soft palate followed by a section of lower thickness that represents the uvula. The cantilever is attached to a rigid wall (hard palate) separating upper (nasal) and lower (oral) inlets of a rigid-walled channel (pharynx) conveying a viscous flow. This model is formulated using the open-source finite-element software library oomph-lib. A parametric study is performed in which the uvula-to-soft palate length and thickness ratios are varied whilst keeping their combined length constant.

Results show that there is a critical uvula-length fraction that determines whether the uvula stabilises or destabilises the system. A relatively 'short' uvula swings out of phase with the soft palate and these oscillations are observed to decay; the mode shapes involved are not predicted by a uniform-thickness plate model. By contrast, if the uvula is relatively 'long' the flexible plate motion is isolated to the uvular section and the oscillations grow in amplitude, indicating a net energy transfer from fluid to structure. Increasing the thickness, hence inertia and flexural rigidity, of a 'short' uvula, e.g., by oedema, makes the fluid-structure system more unstable. In this case if the oedema were aggrevated by the vibratory mechanical insult then it would be self-sustaining and imply a bidirectional relationship between snoring and oedema of the uvula.

Anatomical variability is common in the lengths of the soft palate and uvula which may make some people more susceptible than others to uvulopalatal snoring. Palatal surgery for snoring has proved highly variable in its effectiveness. Modelling of palatal motion using this approach may help guide patient selection for and type of soft-palate surgery applied to treat this common and potentially disabling condition.
\end{abstract}

Keywords: Flutter instability, Beam equation, Soft palate, Uvula. 


\section{INTRODUCTION}

A mechanical analogue to the upper airway is shown schematically in Figure 1(a). It consists of a cantilevered flexible plate attached to a rigid plate immersed in viscous flow through a rigid-walled channel. The FSI of such a system has been demonstrated to capture uvula motion representative of snoring (e.g., Huang et al., 1995; Howell et al., 2009), which is associated with sleep apnoea. Self-excited oscillations of the flexible plate are sustained by a flutter-type aeroelastic instability, which involves an irreversible transfer of energy from fluid to plate. This arises from a phase difference between fluid pressure and plate motion that occurs beyond a critical Reynolds number (Balint and Lucey, 2005; Howell et al., 2009).

The soft palate, although tethered along its sides and leading (anterior) edge, is an elastic plate that participates in inspiratory tissue motion along with its downstream extension, the uvula. Therefore, rather than attaching the uvula to a rigid plate, effectively rendering the soft palate rigid, we approximate the soft palate as a cantilevered flexible plate and at the free end the uvula is represented as a section of reduced mass density, $M^{*}\left(=\rho_{\mathrm{s}}^{*} h^{*}\right)$, and reduced flexural rigidity, $B^{*}\left(=E^{*} h^{* 3} /\left[12\left(1-\nu^{2}\right)\right]\right)$, making the rigid upstream plate the hard palate. Variations in $M^{*}$ and $B^{*}$ are achieved through specifying a thickness profile, $h^{*}$, shown qualitatively in Figure 1(b). As the lengths of the soft palate and uvula vary considerably in both non-snorers and habitual snorers (e.g., standard deviation up to $29 \%$ of the mean value, Reda et al., 1999) the influence of their relative lengths on system stability is investigated in a parametric study.

(a)

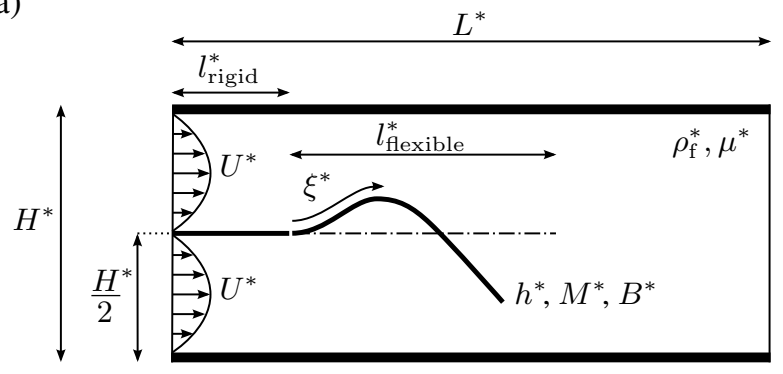

(b)

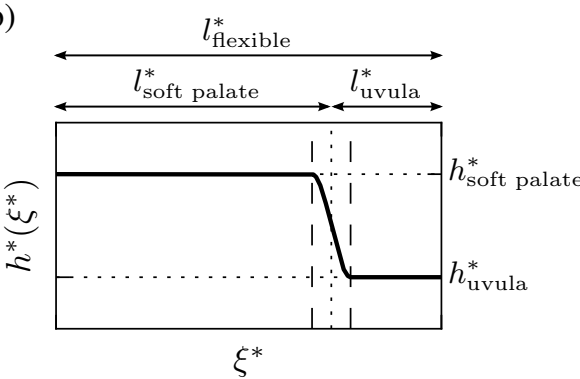

Figure 1. (a) A schematic diagram of the cantilevered flexible plate in viscous channel flow; $h^{*}, M^{*}$, and $B^{*}$ are the flexible plate thickness, mass per unit area and flexural rigidity, respectively; $\rho_{\mathrm{f}}^{*}$ is the fluid density and $\mu^{*}$ its dynamic viscosity. (b) A qualitative plot of flexible plate thickness as a function of the coordinate along the plate, $\xi^{*}$, showing how the uvula is distinguished from the soft palate.

\section{METHOD}

Viscous flow through the 2-D channel is governed by the Navier-Stokes equations, nondimensionalised on the channel height, $H^{*}$, the inflow velocity, $U^{*}$, and the intrinsic fluid time scale, $H^{*} / U^{*}$ (see Figure 1). The flow is then characterised by the Reynolds number $\operatorname{Re}=\rho_{\mathrm{f}}^{*} U^{*} H^{*} / \mu^{*}$. The deformation of the flexible plate is given by the 1-D Kirchoff-Love beam equation, expressed here in nondimensional form via the principle of virtual displacements (PVD),

$$
\int_{0}^{l_{\text {flexible }}}\left[\gamma \delta \gamma+\frac{h^{2}}{12} \kappa \delta \kappa-\left(\frac{1}{h} \sqrt{\frac{A}{a}} T_{i}-\Lambda^{2} \frac{\partial^{2} R_{i}}{\partial t^{2}}\right) \delta R_{i}\right] \mathrm{d} s=0,
$$

where $\gamma$ and $\kappa$ are the respective axial-strain and bending measures, $\sqrt{A / a}$ is the 'extension ratio' of the beam's centerline, and $\mathrm{d} s=\sqrt{a} \mathrm{~d} \xi$ with $\xi$ being the Lagrangian beam coordinate. The nondimensional parameter

$$
\Lambda=U^{*} \sqrt{\frac{\rho_{\mathrm{s}}^{*}}{E_{\mathrm{eff}}^{*}}}
$$


is the ratio of the natural time scale of extensional oscillations in the beam, $H^{*} \sqrt{\rho_{\mathrm{s}}^{*} / E_{\text {eff }}^{*}}$, to the time scale used in the nondimensionalisation of the equations, $H^{*} / U^{*} ; E_{\mathrm{eff}}^{*}=E^{*} /\left(1-\nu^{2}\right)$ is the effective $1-\mathrm{D}$ elastic modulus. The components of the traction vector comprise the summed pressure and viscous loads of the fluid on its 'top' and 'bottom' faces,

$$
\begin{aligned}
T_{i}=Q\{ & {\left[\left.p\right|_{\text {top }} \hat{N}_{i}^{[\text {top }]}-\left.\left(\frac{\partial u_{i}}{\partial x_{j}}+\frac{\partial u_{j}}{\partial x_{i}}\right)\right|_{\text {top }} \hat{N}_{j}^{\text {[top }]}+\right.} \\
& {\left.\left[\left.p\right|_{\text {bottom }} \hat{N}_{i}^{[\text {bottom }]}-\left.\left(\frac{\partial u_{i}}{\partial x_{j}}+\frac{\partial u_{j}}{\partial x_{i}}\right)\right|_{\text {bottom }} \hat{N}_{j}^{[\text {bottom }]}\right]\right\}, }
\end{aligned}
$$

where $\hat{N}_{i}^{[t o p]}$ and $\hat{N}_{i}^{[b o t t o m]}$ are the outer unit normals on the top and bottom faces of the deformed beam. The nondimensional parameter

$$
Q=\frac{\mu^{*} U^{*}}{E_{\mathrm{eff}}^{*} H^{*}}
$$

is the ratio of the viscous pressure scale, $\mu^{*} U^{*} / H^{*}$, used to nondimensionalise the Navier-Stokes equations, to the beam's effective elastic modulus, $E_{\text {eff }}^{*}$, used to nondimensionalise the PVD equation. Thus $Q$ indicates the strength of the fluid-structure interaction and $\Lambda^{2}=\left(\rho_{\mathrm{s}}^{*} / \rho_{\mathrm{f}}^{*}\right) \operatorname{Re} Q$.

In our constitutive law we form the tensor of elastic coefficients with the deformed rather than undeformed metric tensor. This yields a geometrically nonlinear formulation as the strain depends nonlinearly on the displacements, capturing the kinematics of the deformation exactly for arbitrarily large displacements and rotations, though for small strains the difference between this and a linear theory is negligible.

The problem is formulated using the open-source finite-element library oomph-1ib (Heil and Hazel, 2006). For the structural mechanics we use two-node Hermite beam elements and a Newmark timestepper, while for the fluid mechanics we use nine-node quadrilateral Taylor-Hood elements and a BDF timestepper. The FSI problem is discretised monolithically and the Newton-Raphson method is used to solve the nonlinear system of equations, employing the SuperLU direct linear solver within the Newton iteration. The start-up procedure involves (i) applying a distributed load under no-flow conditions to deflect the plate into the second in vacuo eigenmode with small (linear mechanics) amplitude, (ii) constraining the plate in position, (iii) gradually introducing the flow in a series of steady solutions then (iv) unconstraining the plate and solving the unsteady FSI problem. We have tested our code at various mesh densities and timestep sizes, and employed oomph-lib's adaptive mesh refinement capabilities. For further details see Elliott et al. (2010).

The parameter values are summarised in Table 1 and largely follow Tetlow and Lucey's (2009) 'Plate 2 ' model on the premise of predicting representative snoring frequencies in an anamically equivalent 2-D airway. The flexible-plate length is based on soft palate and uvula lengths of $25 \mathrm{~mm}$ and $10 \mathrm{~mm}$ (Reda et al., 1999), respectively, thus necessitating a longer airway to ensure that the downstream exit boundary condition does not influence plate motion. For beams having lengths of $10 \mathrm{~mm}$ and $35 \mathrm{~mm}$, with uniform-thickness and material properties from Table 1, the first four eigenmode frequencies are 4.9, $30.5,85.3,167.1 \mathrm{~Hz}$ and $0.4,2.5,7.0,13.6 \mathrm{~Hz}$, respectively. Snoring frequencies have been measured in range of 20-320 Hz with a mean of $89 \mathrm{~Hz}$ by Brietzke and Mair (2006).

\section{RESULTS \& DISCUSSION}

We first consider the familiar case of a cantilevered flexible plate of uniform thickness. Figure 2 shows a time trace of the vertical position of the tip (free end) for two such flexible plates subject to viscous channel flow, differing only by their length; the $y$ coordinate is normalised by the amplitude of the starting configuration. The oscillatory motion of the $10 \mathrm{~mm}$ plate decays while the relatively slower motion of the $35 \mathrm{~mm}$ plate grows in time. To better appreciate the flutter instability that operates in the $35 \mathrm{~mm}$ case the configuration of the flexible plate over the first oscillation is plotted in Figure 3(b) with the reference in vacuo mode shape for the same initial configuration provided in Figure 3(a). In both plots the initial and final configurations are the bold green and red curves, respectively, and those of intermediate time steps 
Table 1. Dimensional and nondimensional parameter values.

\begin{tabular}{lll}
\hline \hline Parameter & Value & Description \\
\hline$L^{*}$ & $100 \mathrm{~mm}$ & Length of channel \\
$H^{*}$ & $5 \mathrm{~mm}$ & Height of channel \\
$l_{\text {rigid }}^{*}$ & $5 \mathrm{~mm}$ & Length of rigid central wall \\
$l_{\text {flexible }}^{*}$ & $35 \mathrm{~mm}$ & Length of flexible plate \\
$h^{*}$ & $1.75 \mathrm{~mm}$ & Thickness of flexible plate \\
$\rho_{\mathrm{f}}^{*}$ & $1.1774 \mathrm{~kg} / \mathrm{m}^{3}$ & Density of fluid \\
$\mu^{*}$ & $1.98 \times 10^{-5} \mathrm{~kg} /(\mathrm{m} \cdot \mathrm{s})$ & Dynamic viscosity of fluid \\
$M^{*}$ & $0.0248 \mathrm{~kg} / \mathrm{m}^{2}$ & Mass per unit area of solid \\
$B^{*}$ & $1.87 \times 10^{-8} \mathrm{~N} \cdot \mathrm{m}$ & Flexural rigidity of flexible plate \\
$U^{*}$ & $0.2 \mathrm{~m} / \mathrm{s}$ & Mean inlet velocity \\
$\eta_{0}^{*}$ & $0.025 \mathrm{~mm}$ & Amplitude of initial flexible plate eigenmode displacement \\
\hline $\mathrm{Re}$ & 59.5 & Reynolds number \\
$\mathrm{Q}$ & $1.89 \times 10^{-5}$ & FSI parameter \\
$\rho_{\mathrm{s}}^{*} / \rho_{\mathrm{f}}^{*}$ & 842.5 & Solid-to-fluid density ratio \\
$\Lambda^{2}$ & $1.98 \times 10^{-3}$ & Solid-mechanics time scale ratio \\
\hline \hline
\end{tabular}

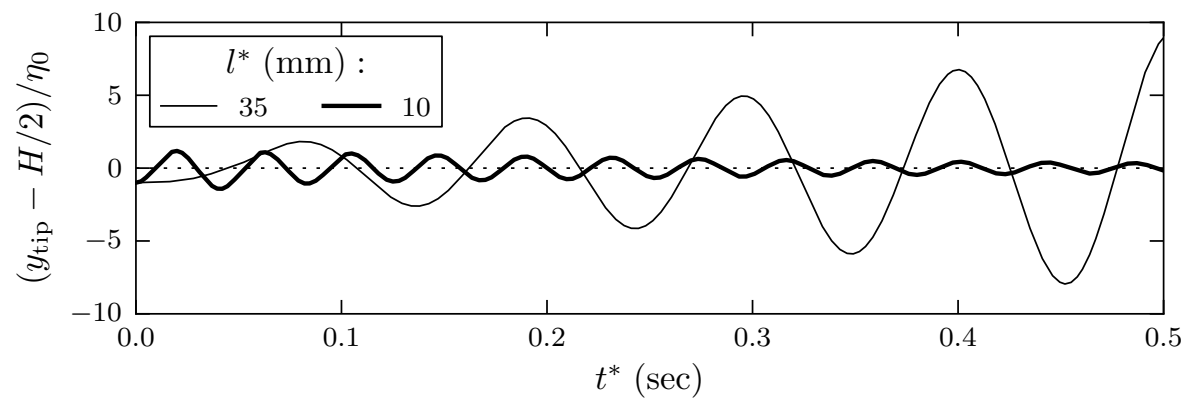

Figure 2. Time traces of span-wise tip deflection for uniform-thickness cantilevered flexible plates of differing lengths $\left(l^{*}\right)$ in viscous channel flow; all other parameter values as per Table 1.

are the thin black curves. The in vacuo oscillation begins and ends in the same configuration describing the second eigenmode [Figure 3(a)]. This single structural mode accounts for the broad geometric features of the unstable flow-induced oscillations seen in Figure 3(b).

The effect of the flexible plate having a nonuniform thickness is shown in the mode-shape plots of Figures 3(c,d). All modelling details are the same as the simulation reported in Figure 3(b) with the exception that the plate thickness tapers from its default value at the clamped end to a quarter of this value at the free end $\left(h_{\mathrm{uvula}} / h_{\text {soft palate }}=0.25\right)$ according to the profile of Figure $1(\mathrm{~b})$, with the two sections being delineated by a vertical dotted line in each plot. The models corresponding to Figure 3(c) and (d) represent the combinations of a $10 \mathrm{~mm}$ uvula and $25 \mathrm{~mm}$ soft palate $\left(l_{\mathrm{uvula}} / l_{\text {flexible }}=2 / 7\right)$ and a $20 \mathrm{~mm}$ uvula and $15 \mathrm{~mm}$ soft palate $\left(l_{\text {uvula }} / l_{\text {flexible }}=4 / 7\right)$, respectively. The mode shape of the $10 \mathrm{~mm}$ uvula model evolves in quite a complicated manner in which higher-order modes are excited, such as modes 3 (red) and 4 (green), clearly visible in Figure 3(c); as a result the uvula moves out of phase to the soft palate. Ultimately, these oscillations die away. In contrast, the model with a proportionally longer uvula exhibits a flutter-type instability. Figure 3(d) depicts the mode shape evolution over a series of oscillations from the initial configuration. It is evident that the flexible plate motion is dominated by mode- 2 oscillations of the uvula section (right of the dotted line) - the soft palate section scarcely participates in the oscillations. In summary, the uvula stabilises the system in Figure 3(c) while the system of Figure 3(d) remains unstable but is marginally less unstable than the homogenous system. 

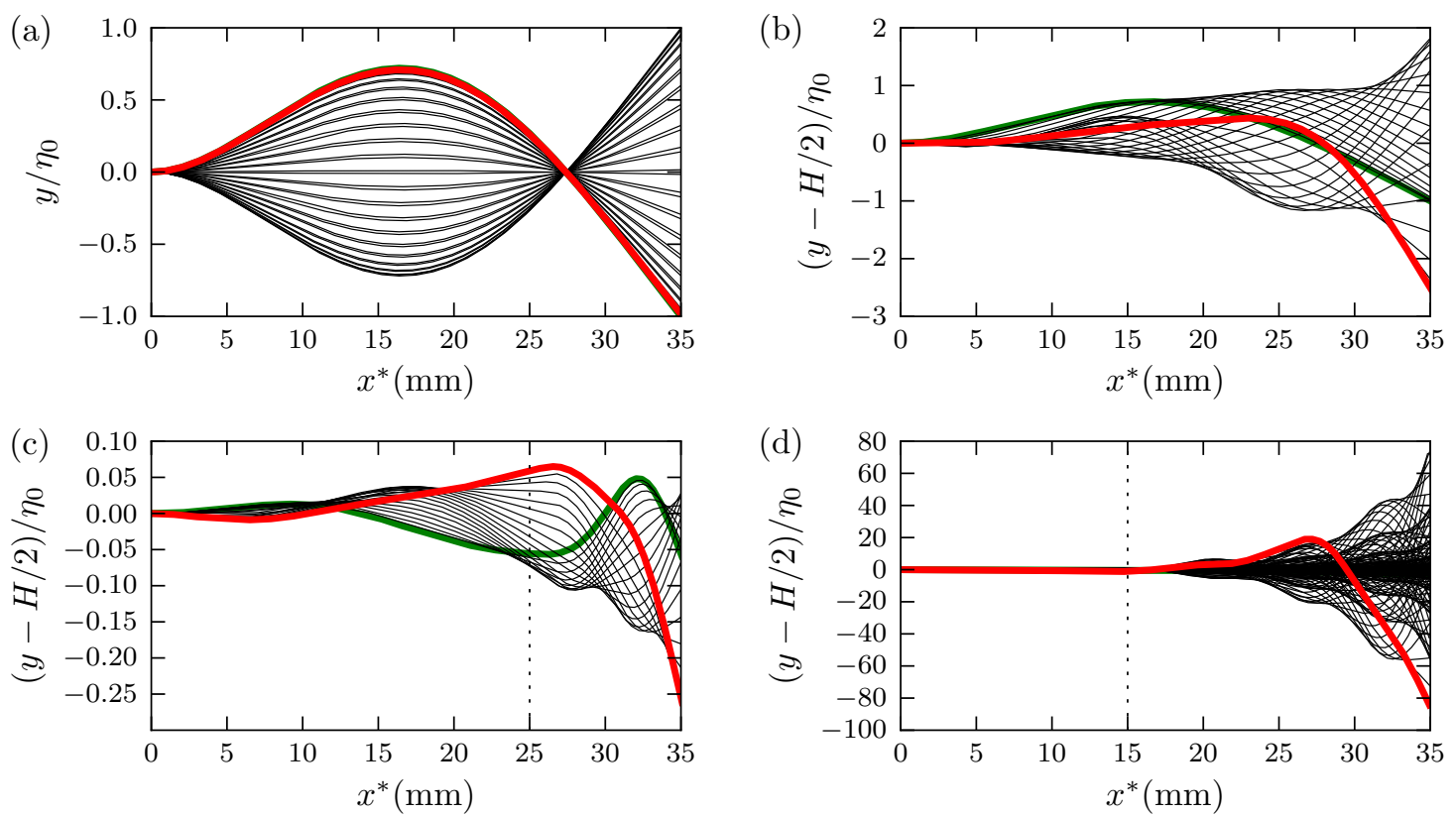

Figure 3. Flexible plate mode shapes for a uniform-thickness plate (a) in vacuo and (b) in viscous channel flow, and for nonuniform-thickness plates having $h_{\text {uvula }} / h_{\text {soft palate }}=0.25$ with (c) $l_{\text {uvula }} / l_{\text {flexible }}=$ $2 / 7$ and (d) $l_{\text {uvula }} / l_{\text {flexible }}=4 / 7$. The bold green and red curves correspond to the first and last time step in each of the sequences plotted. The sub-figures are plotted on different vertical scales and these indicate amplitude growth or decay relative to the scale of unity in (a).

To investigate how system stability is influenced by the length and thickness ratios of the uvula relative to the soft palate we perform a parametric study, which is summarised in Figure 4. Plotted is the growth rate of oscillation, determined from time traces of span-wise tip deflection (i.e., as in Figure 2), for a

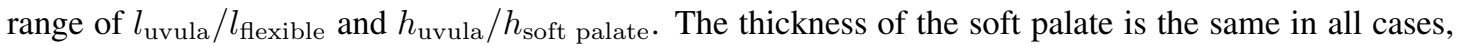
$h_{\text {soft palate }}=h$, and the $l_{\text {uvula }} / l_{\text {flexible }}=7 / 7$ cases correspond to a uniform-thickness flexible plate of

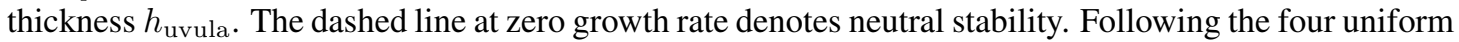
thickness ('7/7') markers from bottom to top it is evident that the system becomes more unstable as the plate thickness is reduced. This is a standard result correlating the flexural restoring forces with system stability (Balint and Lucey, 2005). However, when the flexible plate has a nonuniform thickness the behaviour depends on the length ratio of the two sections, as follows. A long uvula (4/7) shows the same trend for system stability as noted above. For short uvulae (1/7) the foregoing trend is reversed and the system is stabilised by decreasing the thickness of the uvula; the system becomes less unstable than the homogenous case and for further decreases the whole plate-plus-uvula system can become stable. Therefore there exist critical combinations for the values of uvula thickness and length beneath which the uvula-palate system is (i) less unstable that the homogenous system and (ii) stable.

The above counter-intuitive results-that locally decreasing the stiffness can lead to increased system stability - for short uvulae are paralleled in the studies of Howell et al. (2009) and Howell et al. (2011), at least for inviscid flow. The former demonstrated that localised stiffening can be destabilising while the latter demonstrated that inertial inhomogeneity can be either stabilising or destabilising depending on the location of the added mass and the length of the soft palate. These findings have been explained in terms of the spatially varying energy transfers between flow and structure that are the cause of flutter and their disruption through the introduction of structural inhomogeneity. The same effects are also apparent in the re-organisation of the spectrum of fluid-loaded structural modes that inhomogeneity causes.

Finally, in Figures 5(a) and (b) we present a breakdown of the energy in the flexible plate for the nonuniform-thickness cases $\left(h_{\mathrm{uvula}} / h_{\mathrm{soft}}\right.$ palate $\left.=0.25\right)$ plotted earlier in Figures $3(\mathrm{c})$ and $(\mathrm{d})$, respec- 
N.S.J. Elliott et al., Modelling and simulation of fluid-structure interactions in human snoring

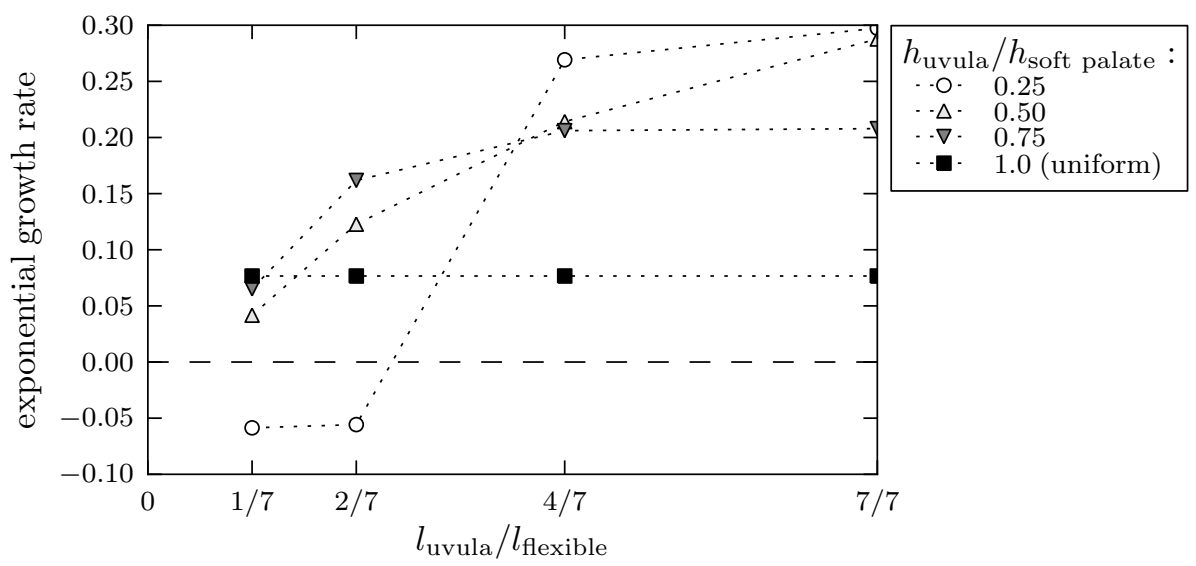

Figure 4. The results of a parametric study showing the influence of the relative dimensions of the uvula and soft palate on system stability; a higher growth rate indicates a more unstable system.

tively. In Figure 5(a), with $l_{\text {uvula }} / l_{\text {flexible }}=2 / 7$, the potential (strain) energy and the kinetic energy of the soft palate and uvula sections are plotted along with their summed total following the release of the flexible plate. The total plate energy oscillates with the greatest contribution initially coming from the cyclic bending of the soft palate section but as the motion decays this gives way to the finite axial strain (potential) energy sustained by the viscous traction as the flexible plate asymptotes to a vertically undeformed shape. Figure 5(b) shows the same energy breakdown for the unstable oscillations of the $l_{\text {uvula }} / l_{\text {flexible }}=4 / 7$ case, with several notable features. The soft palate is essentially stationary so its only energy contribution comes from fluctuating axial strain. The uvula, on the other hand, initially makes a dominant contribution through bending strain (potential energy) but this is eventually surpassed at $t^{*} \approx 0.4 \mathrm{sec}$ by the kinetic energy from its rapid motion. Interestingly, the kinetic energy of the uvula section almost dies out completely $\left(t^{*} \approx 0.2 \mathrm{sec}\right)$ before making its rise associated with the flutter-type instability.

\section{CONCLUSION}

It has been shown that critical combinations of the values of uvula length and thickness exist that demarcate stabilising and destabilising effects of the uvula on flutter of the palate-uvula system. In broad terms, short, thin uvulae are stabilising, moderate-length, thick uvulae are destabilising, and long uvulae, whether thick or thin, are highly destabilising. The more unstable motions of longer uvulae are, by their geometry, additionally likely to cause blockage of the airway and contribute to apnoeic events. Furthermore, some of the mode shapes cannot be predicted by a flexible plate model of uniform thickness. Given the variability in both palatal anatomy and the effectiveness of its surgical augmentation to treat snoring, models that distinguish the soft palate from the hard palate and uvula, such as presented here, may help elucidate the underlying mechanics and improve treatment outcomes.

\section{ACKNOWLEDGEMENT}

We acknowledge the support of the Australian Research Council through project DP0559408 and the WA State Centre of Excellence in eMedicine in the continuing project Airway Tomography Instrumentation.

\section{REFERENCES}

Balint, T. S. and A. D. Lucey (2005). Instability of a cantilevered flexible plate in viscous channel flows. Journal of Fluids and Structures 20, 893-912.

Brietzke, S. E. and E. A. Mair (2006). Acoustical analysis of snoring: can the probability of success be predicted? Otolaryngology-Head and Neck Surgery 135, 417-420. 
Elliott, N. S. J., A. D. Lucey, and M. Heil (2010, 1-5 Aug). Large-amplitude oscillations of a finitethickness cantilevered flexible plate in viscous channel flow. In Proc. ASME 3rd Joint US-European Fluids Engineering Summer Meeting \& 8th International Conference on Nanochannels, Microchannels, and Minichannels, Montreal, Canada. Paper 30348, ISBN 978-0-7918-3880-8.

Heil, M. and A. L. Hazel (2006). oomph-lib - an Object-Oriented Multi-Physics Finite-Element Library. In M. Schafer and H.-J. Bungartz (Eds.), Fluid-Structure Interaction, Lecture Notes on Computational Science and Engineering, pp. 19-49. Springer. oomph-1ib is available as open-source software at: http://www.oomph-lib.org.

Howell, R. M., A. D. Lucey, P. W. Carpenter, and M. W. Pitman (2009). Interaction between a cantilevered-free flexible plate and ideal flow. Journal of Fluids and Structures 25, 544-566.

Howell, R. M., A. D. Lucey, and M. W. Pitman (2011). The effect of inertial inhomogeneity on the flutter of a cantilevered flexible plate. Journal of Fluids and Structures 27(3), 383-393.

Huang, L., S. J. Quinn, P. D. M. Ellis, and J. E. Ffowcs Williams (1995). Biomechanics of snoring. Endeavour 19(3), 96-100.

Reda, M., A. J. Sims, M. Collins, G. J. McKee, H. Marshall, P. J. Kelly, and J. A. Wilson (1999). Morphological assessment of the soft palate in habitual snoring using image analysis. The Laryngoscope 109(10), 1655-1660.

Tetlow, G. A. and A. D. Lucey (2009). Motions of a cantilevered flexible plate in viscous channel flow driven by a constant pressure drop. Communications In Numerical Methods In Engineering 25, 463482.

(a)

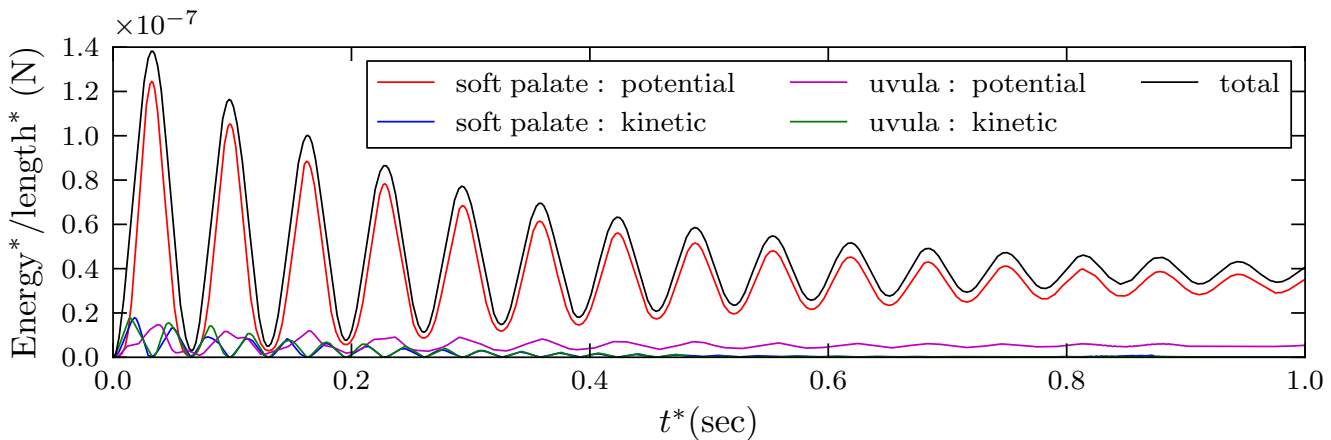

(b)

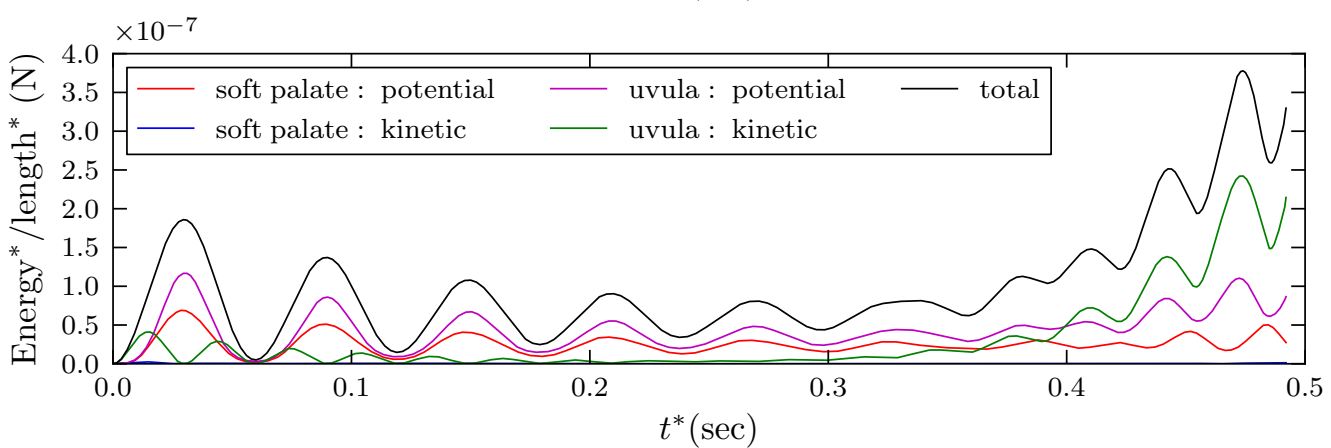

Figure 5. The energy of the flexible plate, broken down into potential (axial and bending strain) and kinetic (motion) components in each of the uvula and soft palate sections, for the (a) decaying oscillations depicted in Figure 3(c) and (b) growing oscillations depicted in Figure 3(d). 